\title{
La educación como espacio de resistencia. Una propuesta desde las Artes y la Educación Social asentada en la pedagogía queer (pedagoqueer)
}

\author{
Irene Escudero Ledesma
}

Investigadora independiente

\section{Resumen}

En el momento actual asistimos a la supervivencia de la institución educativa moderna, asentada en un currículo arcaico y cerrado que genera prácticas educativas y pedagógicas que perpetúan una norma en relación al cuerpo, el deseo y la identidad y reproducen un discurso violento hacia aquella realidad que la subvierte. Por ello, las prácticas educativas deben apostar por nuevos enfoques asentados en la educación posmoderna, transfeminista y queer. Así, desde esta propuesta se aboga por una nueva visión: la pedagoqueer o pedagogía queer. Este nuevo enfoque propone un espacio de resistencia hacia la norma heterocisexista, buscando romperla para ofrecer nuevos modelos de cuerpos, deseos e identidades. Así, se indagará en la conceptualización de la pedagogía queer desde la Educación Social y la Educación Artística como estrategia clave para la ruptura de la heterocisnorma.

Palabras clave: educación; no heterocisnormativa; pedagogía queer; Educación Social; Educación Artística

\begin{abstract}
In this moment we attend to the survival of the modern educational institution, established in an archaic and closed curriculum that generates educational and pedagogical practices which perpetuate a rule related to the body, sexual attraction and identity, and reproduce a violent discourse towards the reality that subverts the rule. Therefore, educative practices have to claim for a new perspective established in a postmodern, transfeminist, and queer education. Hence, this proposal advocates a new view: the pedagoqueer or queer pedagogy. This new perspective proposes a space of resistance against the heterocisexist rule, seeking to break it up in order to introduce new models of bodies, sexual attractions and identities. In this way, the article will investigate the conceptualization of the queer pedagogy, considering the social education and the artistic education as key strategies for the break with the heterocis-rule.
\end{abstract}

Key words: education; no heterocis-rule; queer pedagogy; social education; artistic education 


\section{Introducción}

Antes de indagar en la vinculación de la teoría queer, la educación y la pedagogía, quisiera hacer algunas aclaraciones sobre el enfoque del presente trabajo para contextualizar las aportaciones recogidas. Primeramente, dada mi vinculación con el activismo y la lucha social, he de incluir y tomar como principales referencias las definiciones de la cuestión queer que provienen, precisamente, de estos ámbitos, aunque no por ello se menosprecian las aportaciones académicas. Siendo así, Queer Nation recoge en su manifiesto de 1990, que ser queer significa resistir y que cada día que nos mantenemos vives ${ }^{1}$ supone un acto revolucionario contra los sistemas normativos de poder y opresión. Ser queer es vivir en los márgenes y luchar de forma constante por mantenernos vives junto a nuestres compañeres, amantes, hermanes. Como se apunta en el fanzine Queer Ultraviolence, lo queer no es un lugar estable donde habitar, sino que es la oposición a la estabilidad. El concepto queer es un territorio de tensión donde habitan un discurso y unas prácticas que se posicionan contra toda la narrativa dominante (blanca, capaz, hetero, cis, monógama, etc.) y donde se sitúan personas que a través de su proceso identitario han construido su subjetividad, reconociendo y trabajando sus privilegios. Lo queer no solo se vincula con la sexualidad y el género, sino que supone un total rechazo al régimen normativo en diferentes dimensiones, por ello, se propone la interseccionalidad de la lucha y la ruptura con toda norma que recaiga sobre nuestros cuerpos (clase, raza, género, sexualidad, habilidad, corporalidad...). Además, se propone la articulación de una colectividad más profunda (Queer Ultraviolence). A través del movimiento y el discurso queer se pretende superar la lógica normativa y las relaciones de poder, construyendo nuevos vínculos que sustenten una lucha cotidiana que abra la puerta a la solidaridad y al afecto, posibilitando nuevas formas de deseo -0 no deseo- $y$ de identidad -0 no identidad-.

Centrándonos en el tema que nos atañe, escribir sobre perspectiva queer, educación ${ }^{2}$ y pedagogía queer supone hablar, hoy en día, de espacios de lucha y resistencia contra la lógica normativa por parte de profesionales de la educación, educandes y familias. La cuestión queer atraviesa de forma decisiva la institución educativa, afectando diferentes dimensiones de la misma. Con ello, me refiero a que no solo pone en tela de juicio la cuestión relativa a la deconstrucción de las identidades sexuales y de género, sino que también propone nuevas formas de construir la educación rompiendo con la dicotomía poder-saber (vinculado con el planteamiento de la

${ }^{1}$ El presente texto apuesta por un lenguaje que no reproduzca las estructuras de dominación basadas en el binarismo de género, como propone Carrascal. Por ello, se aboga por el uso de las terminaciones gramaticales en género neutro que comúnmente se realizan con la $-x$. Sin embargo, dado que para determinados sistemas de lectura de texto que emplean personas con diversidad funcional esto es ilegible, en esta ocasión se empleará la terminación -e.

${ }^{2}$ Cuando se habla en el presente texto de educación no solo atañe a la cuestión de la educación formal y reglada, sino que pretende trascender a otras dimensiones educativas, como es la no formal (educación social) e informal (educación familiar y/o comunitaria). A pesar de que el texto se centra más en el ámbito formal de la educación, dada mi experiencia, esto se hace bajo el enfoque de la educación social dent ro de esta estructura. 
educación posmoderna, especialmente con la educación en el arte de Efland, Freedman y Stuh), las prácticas educativas excluyentes y la reproducción del discurso normativo. Por ello, hay que destacar la potencia que tiene lo queer dentro de la educación para todos los agentes implicados en ella. Debemos reiterar que queer significa resistencia en múltiples dimensiones y ruptura de las prácticas y los discursos normalizadores en diferentes esferas. $Y$ para poner en práctica esta resistencia debemos saber elegir nuestras propias estrategias. En este caso, se apela al arte, dentro de la estructura educativa formal, como instrumento para la producción de experiencias artísticas y de conocimiento crítico (Acaso) y la creación de un espacio de no control (Planella), potenciando que les educandes sean capaces de analizar su entorno y sus relaciones para cuestionar su realidad, la cultura, el poder y la norma. Para ello, en esta ocasión hacemos confluir las Artes con la Educación Social, dentro de los centros educativos, para que este conocimiento crítico trascienda a la sociedad y para que les educandes obtengan las herramientas oportunas para la transformación social y su plena participación dentro de esta. Porque, precisamente, la cuestión queer en educación se centra en estas prácticas, en el cuestionamiento de los binarismos dominantes (Louro) y en el tejido de redes y alianzas (Quinlivan y Town).

\section{Supervivencia de la institución educativa moderna ${ }^{3}$}

Asistimos en la actualidad a la supervivencia de la escuela moderna ante la crisis del modelo de Estado de bienestar y la dependencia bilateral de la escuela y el sistema capitalista, lo que implica que el Estado haya perdido su fuerza y haya sido asumido por la potencia del capital, generando una crisis de las instituciones disciplinarias (flores, "El armario").

En lo que atañe al Estado español, la Ley Orgánica 8/2013, de 9 de diciembre, para la mejora de la calidad educativa (LOMCE), supuso que la escuela perdiera parte de su poder al dejar de ser una institución clave, para que el alumnado construyera su futuro y se convirtiera en una parte más del sistema capitalista a través de la formación del alumnado en clave económica (flores "El armario"). Siendo así, la escuela es un aliado más de la estructura capitalista ya que se servirá de esta para segregar los cuerpos en productores y reproductores, los espacios en público-privado, etc. (Solá). Sin embargo, nuestros cuerpos, deseos, relaciones e identidades no encajan en esos roles y esto supondrá un problema de estabilidad para la escuela y para el capitalismo.

Por ello, la vinculación escuela-capitalismo hace que el sistema educativo (en todos sus niveles) se convierta en un mecanismo de control social (Foucault), pues se va a servir de los dispositivos más violentos que tiene para conseguir que el alumnado se encuadre en esta estructura. Así, se articulará una serie de tácticas sustentadas en la legislación educativa vigente vinculadas con el

${ }^{3}$ Cuando se habla de institución educativa o escuela, a pesar de que este texto se centre en las estructuras educativas formales, se pretende abarcar todas sus dimensiones y trasladar estas posibilidades a todas las dimensiones educativas. Con ello, se busca destacar que un posicionamiento queer dentro de la educación es posible y es deseable si se pretende que esta dote de herramientas y posibilidades a les educandes, familias, comunidad, profesorado y profesionales de la educación para que tomen una actitud crítica y desafiante ante la lógica normativa. 
currículo, la práctica educativa o los materiales y libros de texto. Además, también se sirve de aquellas estrategias que se encuentran fuera del marco legislativo, como es la formación y desinformación de les profesionales de la educación, sus experiencias y prejuicios, la infraestructura de los centros, etc. Como tal, la escuela va a ser uno de los principales agentes encargados de reproducir el discurso normativo (en diferentes ámbitos: racismo, clasismo, sexismo, capacitismo, etc.) en toda la comunidad.

Como describe valeria flores ("El armario") la pizarra de las aulas "es un archivo de normas" en la que se repite incesantemente la misma visión del mundo, de los cuerpos y de las relaciones. Sin embargo, podemos encontrar en las fronteras aquellos nombres y realidades que no acceden a formar parte de su tapiz. Estos "designan cuerpos, identidades, deseos, nombres que autodesignan múltiples modos de vivir fugados de la normalidad tan ansiada y (ba)tallada por la institución escolar" (flores, "El armario" s. pág.). En estas fronteras nos encontramos las realidades que habitamos, a saber: los márgenes. Que son los mismos márgenes del libro de texto que rellena una joven bollera con el nombre de la compañera a la que desea. Estos márgenes que habitamos son el territorio (trans)fronterizo desde donde se resiste a la lógica imperante normativa.

"La escuela es una institución heterosexual, heteronormativa y heteronormativizadora" (Warner en Trujillo). La escuela es una institución que reproduce todos los discursos de dominación y poder. Y por ello, si imaginamos y desarrollamos nuevas prácticas y relaciones educativas que rechacen estos discursos estaremos poniendo en peligro la Institución Educativa Moderna. Como profesionales de la educación, familia o comunidad educativa podemos hacer temblar esta estructura educativa arcaica y normativizadora, que promueve unas prácticas excluyentes y unas relaciones de poder desiguales. La educación, entendida como un proceso de construcción de pensamiento crítico, nos exige transformar esta institución, pues solo sirve al capital. Necesitamos prácticas y relaciones educativas antinormativas y transgresoras que formulen nuevos fundamentos pedagógicos, como son la educación posmoderna y la pedagogía queer.

\section{Prácticas educativas y (re)producción del discurso normativo}

La escuela es un mecanismo de control que perpetúa el régimen normativo a través de su discurso y de sus prácticas. Tanto la escuela como las instituciones educativas tienen sus propias estrategias para conseguir que les profesionales de la educación reproduzcan esta norma y que les educandes la asuman de forma (perversamente) "natural". Estas — centrándonos más en el ámbito educativo formal, y concretamente dentro del contexto español- se vinculan con la construcción de un currículo y de unas prácticas educativas excluyentes, amparadas en la LOMCE.

\section{Un currículo arcaico y cerrado}

El currículo, básicamente, son aquellos contenidos que el profesorado debe reproducir y que el alumnado debe asumir como ciertos, sin ser cuestionados por ninguna de las partes porque están incluidos dentro del marco legislativo vigente. Cuando pensamos en el currículo educativo que se 
está desarrollando, especialmente en el Estado español, podemos analizar y comprobar que se trata de un elemento lento, cerrado, hermético, al que el profesorado debe sucumbir.

Por supuesto, los contenidos que se desarrollan en el currículo siven para perpetuar la norma heterocisexista. Como apunta Planella, "si el sujeto se deja modificar sin más, será objeto de instrucción a través de lo que podemos denominar currículum corporal cerrado" (49), estrategia por la cual los cuerpos serán interpelados violentamente cuando se muestren o se expongan públicamente. En el currículo corporal cerrado, los cuerpos se construyen a través del silencio, la norma, la uniformidad, lo físico y la obediencia. A través de determinados contenidos se van a reproducir una serie de discursos normativos y normalizadores que construyen cuerpos uniformados, escapando a cualquier resquicio de disidencia vinculada con la sexualidad, el género, la funcionalidad o la cultura. Pero nuestros cuerpos "han pasado del silencio al grito", son cuerpos que "se han significado, que han hablado" y que "traspasan, cruzan, desestabilizan, rompen con las estructuras que promueven la 'normalización' de las poblaciones" (46). Nuestros cuerpos que gritan, y los que aún permanecen silenciados por las estructuras de dominación, exigen en los espacios educativos nuevas prácticas pedagógicas que posibiliten su existencia (resistencia) en la frontera. Esta es un territorio de lo posible y lo imposible, es un punto de inflexión que nos posibilita la existencia de muchas pedagogías y múltiples corporalidades (Planella).

Necesitamos prácticas pedagógicas transgresoras que se preocupen de las exclusiones que se generan en el espacio de la normalidad y que posibiliten nuevas zonas de identificación y crítica donde poder reconocer las estructuras de dominación y se puedan crear nuevos deseos (Britzman). Así mismo, nace la pedagogía queer o pedagoqueer (Planella y Pie; Planella), que supone un nuevo posicionamiento pedagógico desde donde pueden hablar los cuerpos que escapan a la lógica normativa.

\section{Prácticas docentes excluyentes y normalizadoras}

Nuestro sistema educativo perpetúa las desigualdades a través de intervenciones inadecuadas que, lejos de crear un espacio de crecimiento y capacidad crítica, ha generado un entorno donde se potencia y acentúa la exclusión. Las escuelas y los centros educativos deben responder a los cambios y acontecimientos que se están desarrollando en la sociedad $y$, para ello, se requiere (además de una legislación educativa útil y preocupada por el crecimiento del alumnado) de profesionales de la educación que cuenten con las herramientas precisas. Sin embargo, aun no se encuentran los materiales y las metodologías pertinentes para la formación de les profesionales y la atención a la heterogeneidad de nuestro alumnado. Lejos de ser así, se potencia diluirla y unificar a les educandes bajo un canon cultural (Torres Santomé), que poco obedece al panorama social actual.

Las prácticas educativas se vinculan con múltiples sistemas de opresión y dominación. En vista de ello, se plantea ante nosotres un sistema en el que las violencias se interconectan y tiene como resultado el kyriarcado (Schussler Fiorenza). Con este concepto se pone de manifiesto que existen múltiples estructuras basadas en una concepción jerárquica y de subordinación que, además, están relacionadas entre sí. Por ello, cuando planteamos nuevas prácticas no violentas (no sexistas, no 
racistas) debemos asegurar que no perpetúen otras formas de violencia menos presentes en nuestro imaginario, quizá porque no nos atraviesan de la misma forma, como son el clasismo, el especismo, el capacitismo, la transfobia, etc.

Otra de las prácticas que como profesionales tenemos muy presente es el uso del saber como forma de control. Quien tiene el saber gestiona qué conocimientos se imparten en el aula, eliminando aquellos que considere inútiles o innecesarios. Le profesore que escribe en la pizarra tiene el poder de mostrar y ocultar ciertas realidades a su antojo, según sus intereses, su ideología, sus prejuicios. Pero, como apunta Mérida Jiménez, siguiendo la propuesta de Britzman, el aula/centro puede ser un espacio que potencie el cambio social si las prácticas educativas revisan su estructura autoritaria y se combina con el cuestionamiento cotidiano de la heteronormatividad a través de un aprendizaje transgresor.

Es un hecho que el ámbito educativo aún rechaza al diferente (Trujillo), aún segrega por la capacidad de les educandes, patologiza las identidades, etiqueta de normal/anormal, funcional/disfuncional. La búsqueda de una pedagogía de la tolerancia no hace más que construir "lo diferente" como lo extraño y excepcional, mientras perpetúa la normatividad/normalidad. La pedagogía de la tolerancia exige señalar la diferencia como algo negativo, que debe ser controlado (diferentes aulas, horarios o contenidos). La tolerancia no cuestiona el modelo social de la exclusión sino que lo naturaliza, estableciendo una jerarquía de quienes toleran y quienes deben ser tolerades (flores, "El armario"). Es imprescindible que la institución educativa supere el discurso de la diversidad normalizada y trabaje en la defensa del respeto a las diferencias diferenciadas (Trujillo). Siendo así, se hace más que necesario realizar una propuesta educativa construida desde las teorías feministas, transfeministas y queer.

\section{Educación posmoderna y pedagogía queer. Resistencias dentro del espacio educativo}

Llegando a la cuestión principal del presente texto, pretendo poner de manifiesto la (urgente) necesidad de replantearse las relaciones, la metodología, las prácticas y los contenidos dentro de la educación para transformarla y que sea útil para la ruptura de las desigualdades, la exclusión y las jerarquías culturales, sexuales y de género. Sin embargo, este cuestionamiento no puede hacerse si no se parte desde la fundamentación de las teorías feministas, el transfeminismo y el movimiento queer. Teniendo presente este punto de partida, propongo la reformulación de la educación para la deconstrucción del binarismo sexual y de género y las jerarquías culturales bajo el enclave de la educación posmoderna (Efland, Freedman y Stuh) y la pedagogía queer o pedagoqueer (Planella y Pie; Planella). Pero este posicionamiento va más allá de incluir la cuestión de la sexualidad no normativa dentro del currículo y exige un cuestionamiento de la noción de correcto/incorrecto, normal/anormal, que retuerzan los límites del conocimiento socialmente construido y dominante (flores, 2009). Esta aplicación será posible, dentro del contexto español y la aplicación de la LOMCE, a través del desarrollo de las competencias artístico-cultural y cívico-social, especialmente en la etapa de Educación Secundaria, pudiéndose extrapolar a otras. Vinculando ambas competencias para el desarrollo de la personalidad, la autonomía y la capacidad crítica y reflexiva de les educandes para el ejercicio de la libertad. 


\section{Educación posmoderna: punto de partida}

Como destaca Lopes Louro ("Os estudos queer"), el campo educativo es un ámbito especialmente complicado ya que, históricamente, ha sido una institución de la disciplina y se ha comprometido con la integración pero no con la transgresión o la transformación. Continúa apuntando que dentro de esta la subversión no ha sido siempre bien recibida y que por ello nos encontramos ante la necesidad de renovar la educación. El crítico propone reinventar la educación desde la posmodernidad. Este planteamiento procura romper con la noción de centro y pone la mirada en los márgenes, en las diferencias, disponiéndose a cuestionar las fronteras.

Aproximándonos, en una pincelada que se ampliará posteriormente, a la educación posmoderna, bajo el enfoque de la enseñanza artística de Efland, Freedman y Suth, su objetivo principal es conseguir que les educandes sean capaces de comprender "los mundos sociales y culturales en los que viven" (125) a través de la producción de experiencias artísticas que posibilitan el conocimiento crítico (Acaso) y mediante el cuestionamiento de las relaciones de poder y de conocimiento para proponer un nuevo posicionamiento.

Uno de los puntos clave de este planteamiento es la cuestión del poder y el conocimiento, saber que quien tiene el poder tiene el conocimiento, y viceversa. $\mathrm{Y}$, siendo sinceres, esta premisa se repite constantemente en nuestros centros a través de las lecciones del profesorado y los libros de texto que repiten incesantemente la misma versión del conocimiento y de la cultura, sin duda, hegemónica. Igualmente, aparece el concepto de "deconstrucción", que, vinculado a la educación, potenciará la ruptura con las prácticas educativas y las cuestiones normativas, con el fin de desnaturalizar estos procesos y ofrecer alternativas sustentadas en la libertad, el descubrimiento personal y el crecimiento social desde la intervención educativa y, en este caso, artística.

En relación al binomio poder-saber se plantea el interrogante sobre el peso del aprendizaje de la cultura dominante y del protagonismo de les educandes dentro de su proceso educativo. Con ello, la educación posmoderna reivindica un cambio educativo que promueva una nueva cultura crítica, democrática y de carácter social, que atienda las diferentes voces existentes en nuestra comunidad. Por ello, en el proceso educativo y la deconstrucción de la cultura hegemónica, se postula una línea de enseñanza-aprendizaje abierta a aquellas que no corresponden con la idea hegemónica (Aronowitz y Giroux en Efland, Freedman y Stuh), posibilitando la irrupción en el conocimiento de los grupos no hegemónicos y poniéndolo en valor.

\section{Pedagogía queer o pedagoqueer: nuestra resistencia}

Queer se vincula con un conjunto de saberes y una disposición política (Lopes Louro). Vincular lo queer con la educación implica poner en manifiesto los cuestionamientos de la ruptura de los binarismos dominantes y la construcción de alianzas (Quinlivan; Town). Ello supone una posición de resistencia ante la lógica dominante y hegemónica normativa y normalizadora.

Siendo así, parece obvio e imprescindible mantener una mirada queer dentro de la educación, que como apuntan Berná, Cascone y Platero, "se resuelve en una práctica queer" (11). Sin embargo, la realidad y la acción queer van más allá proponiendo un nuevo enfoque metodológico y 
pedagógico, ya que contestan a la normalización pedagógica y cuestionan los recursos educativos construidos bajo la lógica heterosexista (Planella y Pie). Ante este nuevo enfoque se construye la pedagogía queer o pedagoqueer.

Plantear una pedagogía transgresora supone preocuparse por la exclusión que genera la normatividad y construir espacios alternativos para reconocer críticamente las estructuras de dominación creando así mismo nuevos deseos e identidades (Britzman). La pedagogía queer posibilita la proliferación de infinidad de nuevas identidades (Trujillo) en las que toda la comunidad educativa puede indagar. En definitiva, esta propuesta pedagógica suma posibilidades a la educación para que sea transgresora y rompa con los discursos normativos y normativizadores a fin de potenciar el crecimiento libre y el conocimiento crítico sobre la realidad que nos rodea.

Por ello, si queremos una educación que verdaderamente se preocupe por construir estos espacios de pensamiento crítico, es necesario que la vinculemos con la cuestión queer como motor de acción para la transformación social. Si se quiere repensar la educación, Trujillo apunta que la pedagogía queer es un puerto interesante en el que desembarcar.

\section{La pedagogía queer como base de la educación social y la educación artística. Una propuesta educativa}

Tras este análisis y conceptualización de la teoría queer dentro de la educación y su desarrollo como pedagogía queer o pedagoqueer, nos encontramos con la cuestión de cuáles son las implicaciones prácticas que se pueden desarrollar dentro del espacio educativo. En esta ocasión y dada mi propia experiencia, se ha apostado por el desarrollo de la Educación Social y la Educación Artística bajo el enclave de la pedagogía queer, dentro de las competencias artístico-cultural y cívico-social que prevé la LOMCE para la educación secundaria. Esta elección se debe a las posibilidades que tienen ambas ramas educativas desde una lectura queer dentro de la estructura educativa formal, ya que potencia su valor.

Se apuesta por el desarrollo de una Educación Artística porque posibilita que el alumnado adquiera una nueva percepción del otre, de une misme y de su entorno. Por ello, mediante una experiencia de encuentro a través del arte se pretende que la persona tome una posición crítica ante la realidad que la rodea y quiera imaginársela de otra forma (Moreno González). Se reivindica así la idea de que el arte incide no solo en el crecimiento personal sino en la transformación social, principio clave en el que confluye con la Educación Social. Entendemos que el arte es la herramienta fundamental para establecer "itinerarios para la transformación y el cambio, que promocionen además la integración social de los grupos más vulnerables o en riesgo de exclusión" (Guerrero 7). Vinculando Educación Social y Educación Artística parece obvio e imprescindible hacerlo desde la perspectiva queer para que esta práctica educativa persiga la transformación social y el desarrollo del pensamiento crítico a fin de rechazar y romper con los sistemas de opresión, dominación y normatividad que imperan dentro de nuestra sociedad, del espacio educativo y de la comunidad. 
Como apuntan Huerta y Alonso-Sanz, el arte es un ámbito de conocimiento que se pone al servicio de la defensa de las minorías, la reivindicación por los derechos y la visibilización de los colectivos y las culturas no hegemónicas. Así, nos interesa destacar brevemente tres experiencias dentro de la Educación Artística para su desarrollo dentro del espacio educativo que pueden ser herramientas clave en el crecimiento de les educandes, el cuestionamiento de les profesionales y la transformación social en clave queer.

La primera es el arte relacional que aparece de la mano de Nicolas Bourriaud. En esta experiencia artística se busca el encuentro con el público (educandes) a través del desarrollo de experiencias artísticas no tradicionales y de duración abierta e ilimitada (Bourriaud en Costa). Con ello se reclama que el público participe de la creación artística a fin de lograr un intercambio de experiencias para la transformación social y el desarrollo de una perspectiva crítica. Se pone el énfasis en la participación y el intercambio, sintiéndose les alumnes parte de esta experiencia artística donde sus aportaciones son válidas e imprescindibles para este encuentro artístico.

La siguiente se basa en el desarrollo de la educación artística posmoderna de Efland, Freedman y Stuh, que busca el aprendizaje a través de la experiencia. Este nuevo paradigma pretende romper con la dicotomía poder-saber y propone a les educandes un nuevo espacio dentro de su aprendizaje como protagonistas del mismo. La enseñanza basada en el arte procura que les educandes sean capaces de entender el mundo social y cultural en el que viven (Efland, Freedman y Stuh) a través de la producción de experiencias artísticas y de conocimiento crítico (Acaso).

Y la última propuesta se basa en una experiencia de intercambio a través de la narrativa visual, como es el foto-diálogo. Esta técnica intenta establecer un diálogo usando imágenes como medio de comunicación, ya que estas tienen la capacidad de presentar e interpretar lo representado, posibilitando la formulación de preguntas, la defensa de posiciones éticas y el desarrollo de conclusiones justificadas (Roldán en Mora García). El objetivo de esta propuesta didáctica es que les educandes comprendan e interpreten visualmente su realidad haciendo preguntas y dando respuestas en imágenes. Gracias a este método se pretende no solo propiciar el pensamiento artístico y visual, sino también potenciar las habilidades sociales y comunicativas del alumnado mediante el desarrollo del diálogo con le otre y la transmisión de sus inquietudes y necesidades con el uso de la imagen (Mora García).

Vinculando las propuestas mencionadas con la educación social, que tiene cabida dentro de la estructura educativa formal, debemos entender que esta es un derecho de la ciudadanía y que posibilita la creación de contextos educativos y acciones mediadoras y formativas que favorecen la incorporación del sujeto de la educación a diferentes redes sociales, a fin de ampliar sus perspectivas laborales, educativas o de participación (ASEDES y CGCEES, 2007). La educación social es una prestación educativa que se debe al cumplimiento de los principios fundamentales de justicia y desarrollo democrático. Siendo así, este ámbito educativo persigue la transformación social y la participación ciudadana de los sujetos de la educación para su empoderamiento y crecimiento en diferentes esferas de su vida. 
Todo ello solo es posible si se adopta un enfoque queer de la educación. Es decir, no se puede alcanzar los objetivos de la educación, vinculados con el crecimiento personal, académico y social de les educandes, si no es a través de la reformulación de nuestras prácticas educativas o docentes. $Y$, sin duda, esta reformulación debe encaminarnos a la ruptura con los sistemas de dominación y opresión, al rechazo del discurso normativo dentro de la escuela y a la oposición frente a las prácticas educativas excluyentes. Por ello, en esta ocasión, entendemos que la educación social y la educación artística son las herramientas clave a desarrollar bajo el enfoque de la pedagogía queer si pretendemos que la educación cumpla con su verdadero fin.

Pero, ¿cómo trasladamos estas propuestas a nuestra práctica educativa? Este texto no pretende resolver con prácticas concretas sobre qué se debe hacer, pues procura, más bien, generar un territorio de tensión y de cuestionamiento. Por ello, la clave es empezar por une misme, por nuestros propios discursos, prácticas y vivencias. Una práctica educativa queer comienza en la revisión y reformulación de nuestras prácticas educativas, de nuestra identidad y nuestras relaciones. La transgresión llega a las aulas cuando rompemos las jerarquías de poder y dejamos que les educandes, las familias y la comunidad se impliquen en el proceso educativo. Lo queer llega y se asienta en la educación cuando desnaturalizamos el poder y los discursos de dominación y proponemos el desarrollo de la autonomía y la libertad. E implica promover, junto con les educandes, familias, comunidad y otres profesionales la transformación social. Colectivizar conocimientos y valorar las experiencias de le otre. Disfrutar de aprender, de conocer, de equivocarse y de crear. $Y$, finalmente, retorcerse del placer al sentir que se educa y se experimenta la libertad propia y ajena dentro del conocimiento crítico, la solidaridad y el encuentro con le otre.

\section{Breves conclusiones}

Las conclusiones que se extraen de este texto ponen de manifiesto la decadencia de la Institución Educativa Moderna como sistema de dominación vinculado con el capitalismo si se plantean nuevas propuestas educativas y se reformulan las relaciones educativas existentes dentro de los centros. Todo ello debe hacerse desde una mirada queer de ruptura con los binarismos sexuales y de género y la cultura hegemónica, así como con otras estructuras de dominación.

Asimismo, se entiende que la presencia de la cuestión queer dentro de la educación supone un acto de resistencia tanto para les profesionales como para educandes y familia. El impulso queer potencia producir y deconstruir discurso, cuestionar las estructuras de dominación y rechazar las prácticas educativas excluyentes. Mantenerse vive siendo queer dentro del ámbito educativo actual supone un verdadero acto revolucionario ya que la Institución tiene todo un dispositivo de disuasión encargado de reprimir, obstaculizar, penalizar y martirizar este posicionamiento de género, sexual, corporal e identitario. Por ello, si extendemos la cuestión queer e invadimos la educación, conseguiremos que la Escuela Moderna, como institución represiva, y su vinculación con el sistema capitalista, entre en decadencia y sea necesaria su reformulación desde dentro. 
Ante esta situación se hace muy necesario reformular estas prácticas, contenidos y metodologías desde un enfoque feminista, transfeminista y queer. Por ello, se propone un nuevo enfoque construido en clave de la educación posmoderna y la pedagogía queer o pedagoqueer. Desde este posicionamiento no solo se pretende romper con las cuestiones vinculadas con el binarismo de género y sexual, sino que se reformulan los contenidos y las relaciones que se establecen dentro del espacio educativo para que la educación sea un territorio libre de mecanismos de control, de discursos represivos y de sistemas normativos.

Sembrar el fundamento queer en nuestras aulas posibilitará la aparición de una educación que dinamite los sistemas de opresión y represivos y facilite que les educandes tengan las herramientas precisas para transformar sus relaciones y su comunidad. Tenemos las estrategias, ahora podemos crear nuestra resistencia queer en la educación.

\section{Referencias bibliográficas}

Acaso, María. rEDUvolution: hacer la revolución en la Educación. Barcelona: Paidós, 2013. Impreso.

ASEDES y CGCEES. Documentos Profesionalizadores: definición de Educación Social, Código Deontológico del educador y educadora social, Catálogo de funciones y Competencias de la educadora y el educador social. Barcelona: ASEDES, 2007. Impreso.

Berná, David, Michele Cascone y Raquel (Lucas) Platero. “¿Qué puede aportar una mirada queer a la educación? Un estado de la cuestión sobre los estudios sobre LGTBfobia y educación en el Estado español". The Scientific Journal of Humanistic Studies 6 (4) (2012). Web. 28 oct. 2016 $<$ https://goo.gl/xxYf5f >.

Blanco, Jessie. "El cuerpo como discurso de resistencia: subjetividad, cuerpo y práctica contrahegemónica desde una mirada feminista del transgenerismo". Caracas: Universidad Central de Venezuela. Web. 1 nov. 2016 <https://goo.gl/waiU7N>.

Britzman, Deborah. "Is there a queer pedagogy? Or, stop reading straight". Educational Theory 45.2 (1995): 151-65. Impreso.

Butler, Judith. El género en disputa. El feminismo y la subversión de la identidad. Trad. M. ${ }^{\text {a }}$ Antonia Muñoz. Buenos Aires: Paidós, 2001. Impreso.

Carrascal Tris, Sofía. La Educación Social como prevención de la trans`fobia en la infancia. Valladolid: Universidad de Valladolid, 2015. Web. 30 oct. 2016 <https://goo.gl/tEbEjg>. Trabajo de fin de grado.

Costa, Flavia. "De qué hablamos cuando hablamos de 'arte relacional'". Ramona. Revista de artes visuales 88 (2009): 9-17. Impreso.

Dewey, John. El arte como experiencia. Trad. Jordi Claramonte. Barcelona: Paidós, 2008. Impreso.

Efland, Arthur, Freedman, Kerry y Stuh, Patricia. La educación en el arte posmoderno. Trad. Lucas Vermal. Barcelona: Paidós, 2003. Impreso. 
flores, valeria. "El armario de la maestra tortillera. Políticas corporales y sexuales en la enseñanza". escritos herèticos 3 jul. 2009. Web. 4 nov. 2016 <https://goo.gl/bjV4qv>.

. "Entre borrados y tizas. La escritura de los cuerpos y la gramática heteronormativa". escritos herèticos 4 sep. 2011. Web. 4 nov. 2016 <https://goo.gl/vcqf1h>.

Foucault, Michel. Vigilar y castigar: Nacimiento de la prisión. Trad. Aurelio Garzón del Camino. Buenos Aires: Siglo XXI, 1976. Impreso.

Guerrero, Catalina. "Arte, Mayores y Discapacidad. Arte diverso para capacidades diversas". Alfabetizaciones, transalfabetizaciones e inteligencias múltiples: el componente transversal en el aprendizaje y las pedagogías críticas. Eds. Marín Pérez et al. Murcia: Universidad de Murcia, 2015. 1-18. Impreso.

Huerta, Ricard. "La educación artística como motor de cambio social". Cuadernos de Pedagogía 449 (2014): 48-50. Web. 3 mayo 2017 <https://goo.gl/iJ34V3>.

Huerta Ricard y Amparo Alonso-Sanz, eds. Educación artística y diversidad sexual. Valencia: Universitat de València, 2015. Impreso.

Jefatura del Estado. Ley Orgánica 8/2013, de 9 de diciembre, para la mejora de la calidad educativa (LOMCE). Jefatura del Estado: 2013.

Lopes Louro, Guacira. "Os estudos queer e a educação no Brasil: articulações, tensões, resistências". Contemporánea 2.2 (2012): 363-9. Web. 28 oct. 2016 <https://goo.gl/C7dDKr>.

Mérida Jiménez, Rafael M., ed. "Prólogo." Sexualidades transgresoras. Una antología de estudios queer. Barcelona: Icaria, 2002. 7-25. Impreso.

Mora García, Amalia. "El FotoDiálogo como instrumento didáctico de enseñanza, comunicación y desarrollo personal e interpersonal: análisis de estado y propuestas de una web de diálogo por medio de arte". Educación Artística. Revista de Investigación 4 (2013): 251-64. Web 28 oct. 2016 <https://goo.gl/oVX7LX>.

Moreno González, Ascensión. "La mediación artística. Un modelo de educación artística para la intervención Social a través del arte". Revista Iberoamericana de Educación 52.2 (2010). Web. 28 oct. 2016 <https://goo.gl/WiZqy9>.

Planella, Jordi, "De cuerpos, carnes y pedagogías. Travesías corporales en la educación actual". Educación Artística y Diversidad Sexual. Eds. Ricard Huerta y Amparo Alonso-Sanz. Valencia: Universitat de València, 2015.43-59. Impreso.

Planella, Jordi y Asun Pie. "Pedagoqueer: resistencias y subversiones educativas". Educación XX1 15.1 (2012): 265-83. Web. 3 nov. 2016 <https://goo.gl/qb7ZAH>.

Queer Nation. "Queers Read This". Taller de Teoría Queer 28 mayo 2013. Web. 4 nov. 2016 $<$ https://goo.gl/Ktllf4>.

Queer Ultraviolence. Fanzine. 10 feb. 2013. Web. 3 mayo 2017 <https://goo.gl/a0oCIP>.

Quinlivan, Kathleen y Shane Town. "Queer pedagogy, educational practice and lesbian and gay youth". International Journal of Qualitative Studies in Education 12.5 (1999): 509-24. Web. 25 oct. 2016 <https://goo.gl/XItXmG>.

Riot, Coco. Llueven queers. Montreal: Coco Riot, 2010. Impreso.

Schussler Fiorenza, Elisabeth. Los caminos de la sabiduría: una introducción a la interpretación feminista de la Biblia. Trad. José Manuel Lozano Gotor. Maliaño: Sal Terrae, 2001. Impreso. 
Solá, Miriam, ed. Transfeminismos. Epistemes, fricciones y flujos. Tafalla: Txalaparta, 2013. Impreso.

Torres Santomé, Jurjo. La justicia curricular. El caballo de Troya de la cultura escolar. Madrid: Morata, 2010. Impreso.

Trujillo, Gracia. "Pensar desde otro lugar, pensar lo impensable: hacia una pedagogía queer". Educ. Pesqui 41 (2015): 1527-40. Web. 28 oct. 2016 <https://goo.gl/lcwa33>. 